\title{
Editorial: \\ New Models of Aging: Comment on Need and Creative Efforts
}

In this special issue there are some new thoughts about aging that deserve reading. The papers and commentaries are written by authors with experience in research and scholarship who have a serious interest in understanding aging. The material was prepared in response to a developing perspective that the study of aging has become a field of knowledge that is data rich and theory poor, a vast collection of unintegrated pieces of information. As early simplistic single variable explanations of aging were discarded, their place was filled by microgerontology consisting of "aspect research" and microtheory. In response to this perspective, a symposium was organized on Models of Aging for the International Congress of Gerontology held in Budapest in July 1993. Deliberately, four different points of view were sought: biological, psychological, social, and epidemiological-ecological.

The symposium speakers were asked to discuss models of aging from their own disciplinary orientation, to state their preferences and perhaps present their own creative efforts to advance the sophistication of our models of aging. The papers were submitted to a review process and are published in this issue of the Canadian Journal on Aging along with commentaries and rejoinders.

This special issue is devoted to the search for more effective concepts and models of aging. The papers are designed to help us design and carry out more effective research leading to greater understanding of the processes of aging and thereby provide a basis for the amelioration of deteriorative conditions.

I find the papers and the commentaries stimulating and I hope they will be widely read. I also hope they will encourage others to think seriously about the implicit and explicit models that the authors use in explaining aging. There seems little purpose for me to add detailed commentary in this issue but I would like to mention a few points.

Historically, we seem to have adapted our concepts and models from areas of our disciplines that were not oriented to explaining change. Most early biology, psychology and sociology was based on static properties. Our scientific backgrounds have not helped us much in explaining how cells differentiate and undergo senescence, how individuals are not only a product of social structure but determine structure, and how the environment and learning modify the expression of the genome. Clearly, we have to face evidence that there must be important interactive forces in aging, e.g., the large associations between morbidity and mortality and social class. But this comment does not address the unanswered question of what are the dynamic processes. Social structures are not alive, economies are not alive, organisms 
are. Organisms are self-structuring energetic systems, unlike environments.

These papers lead me to believe that we have had limitations in modelling aging because the outcomes appear to be a complex outcome of interactions of self-structuring organisms with their environments. Models of aging seem to require concepts of both energy and information. In the papers we read terms like entropy, negentropy, and gerodynamics. These terms seem to locate the forces of aging in biological processes. Yet I cannot easily classify all of the variance solely as biological if I learn that in this century about 30 years has been added to average life expectancy or that arctic tribal people moving to urban areas show altered patterns in their physiological signs of aging.

My reading of the material leads me to view aging as an ecological process, an interaction of organisms with particular genetic backgrounds with particular social and physical environments. The search for the suspected interactions leads me to anticipate that we will begin to identify subpatterns of aging, "effective causal complexes," as one writer terms them.

It is questionable whether we will want to adopt single clock models of aging, whether biological, psychological or social in view of the discontinuities suggested in some of the writing. Yet many aspects of living systems show rhythmic activity. Continuity and discontinuity, stability and change, differentiation and dedifferentiation are among the terms that fit some features of aging. There are some other less familiar terms used in these discussions that may replace earlier expressions as we improve our discourse and grasp of the organization of aging as a natural phenomenon.

The exchanges between the authors and the reviewers suggests that improvements in our models and theories depend upon the kinds of questions we ask of our data and the methods used to answer them. Future comprehensive and sequential longitudinal studies will help separate influences on aging into finer categories of effects resulting from secular drifts in populations and cohort differences, time of measurements, and organism properties. One might think ahead to partitioning the variance in how we age, based not only on social structural and genetic characteristics but on the effects of fetal life carried forward into adulthood. For example, light weight babies are at risk and tend to be born of young unmarried women of low socio-economic status. Are there late life outcomes of such early life conditions?

Understanding aging is a very complex task. The processes of aging appear to be one of the most complex subjects to challenge scientific study and explanation. This may explain our apparent willingness to study limited aspects of human aging and generate micro theories. In response to this state of affairs these papers are intended to provoke more integrative thought, to link the aspects into larger explanatory theoretical perspectives.

The field of study has moved away from early attempts to use single variable explanations of aging, from views that we had a given number of heart beats to be used up quickly or slowly, or that aging was the result of gastroin- 
testinal putrefaction or a result of the action of a single hormone that was prepotent in controlling our length of life. In rejecting the simple we seem to have stalled in developing more complex explanations. A reasonable person would likely subscribe to the idea that human aging is a result of the genome interacting with the circumstances of the physical and social environments. The dramatic gains in life expectancy in this century, not only at birth but at advanced age as well, suggest that aging is an example of ecological relationships whose outcomes shift as new circumstances arise.

There is no particular order in which to read these papers and their commentaries. I suggest you begin with a topic close to your interests and then go on to the others. The papers are systematic efforts by recognized scholars to deal with the complexities and perplexities of aging. When you have read them a question for you to answer is, "What is my model of aging?" I believe this collection of thoughts will help all of us to refine the models of aging that we use in planning our research and teaching, and organizing services to aging individuals and aging societies.

I am proud to have had the opportunity to organize the symposium on models of aging that led to this publication. I appreciate the efforts of the participants and the reviewers for their commentaries. The editor, François Béland, must be thanked for his interest and support of this publication. This special issue of the Journal will be read in many countries by senior scholars, students and professionals who want to understand aging, one of the least understood features of our existence.

I hope that readers will want to join me in thanking the authors for their work in preparing the papers and the reviewers for their thoughtful comments as well as the rejoinders by the authors. Not only do we have sound thinking presented in this issue but as well we have lively criticism, the essence of a productive scientific forum. The Editor has done us a service by encouraging publication of this attempt to strengthen our thinking about a very complex and important subject, aging. 